\title{
On the Photoionization of Large Molecules
}

\author{
C. H. Becker and K. J. Wu* \\ Molecular Physics Laboratory, SRI International, Menlo Park, California, USA
}

\begin{abstract}
There is no apparent limit to the size of a molecule for which photoionization can occur. It is argued that it is difficult to obtain useful photoionization mass spectra of peptides (above $\sim 2000 \mathrm{u}$ ), proteins, and oligonucleotides, because of the high internal energy of these polar molecules as a result of the desorption event and because vibrationally excited radical cations readily fragment. Evidence to support this hypothesis is presented from the 118-nm single-photon ionization (SPI) mass spectra of the cyclic decapeptide gramicidin $S$ and of fullerenes, from null SPI results with the linear peptides substance P and gramicidin D and oligonucleotides, and from a variety of data found in the literature. The literature data include mass spectra from jet-cooled peptides, perfluorinated polyethers, collisional ionization of small neutral peptides, and the ultraviolet photoelectron spectroscopy of polymeric solids. (I Am Soc Mass Spectrom 1995, 6, 883-888)
\end{abstract}

$\mathrm{M}$ uch recent effort in mass spectrometry has been directed toward the analysis of peptides and proteins $[1,2]$, DNA $[3,4]$, and other large biopolymers. Successful analytical approaches to date have used the direct ionization associated with the desorption process, especially with laser desorption. In contrast, attempts at photoionization (PI) of gaseous biomolecules of large size $(\geq 1000 \mathrm{u})$ have met with only limited success [5-8]. The largest peptides photoionized reproducibly were $\leqslant 2000$ u using jet cooling after laser desorption and before PI $[5,6]$.

Recent arguments state that the lack of effective PI for larger molecules is due to charge-pair formation where recombination dominates over PI $[9,10]$. This has been described in terms such as the large isolated molecule acts as its own solvent--the high density of states associated with large molecules. In this paper it is proposed that, at least for the case of biomolecular oligomers, the inability to observe large photoions is not related to the ability to cause photoionization, but rather is associated with efficient photofragmentation of the radical cation formed. This is not to say that there is not a channel where fragmentation occurs that leads to only neutral products, but rather that a channel with an ionized fragmented product also is substantial.

Peptides are generally highly polar molecules that are capable of substantial hydrogen bonding. Except for most amino acids and very small peptides, they do not desorb intact under slow thermal heating. Desorption then requires some rapid process. It is well known that for the case of matrix-assisted laser desorption

\footnotetext{
Address reprint requests to Dr. ( H. Becker. Molecular Physic Labotatory, SRI International, Menk I'arh, CA 94125.

* Present address: Charles Evans and sissociates. 301 Chesapeake Drive, Redwood City, CA 94063.
}

(MALD), peptides and proteins show significant degrees of metastable decay that increases with the mass of the molecule [11]. Even for the MALD technique, considered currently to be the "gentlest" of all stimulated desorption techniques in that it permits observation of the quasimolecular ions of large proteins and DNA oligomers, much energy is required to desorb large molecules as evidenced by this metastability. We hypothesize that the difficulty in obtaining radical photoions of these large molecules is due to their increasing internal energy per bond (not effectively cooled for the larger molecules even by exposure to a supersonic jet after desorption) and the lower stability of the radical ion, relative to the closed-shell ion, which leads to efficient bond cleavage. Evidence in support of this hypothesis is presented.

\section{Experimental Details}

We examined by laser desorption the peptides gramicidin $S$ and $D$ and substance $P$, as well as small ( 3000 u) single-stranded mixed-base oligonucleotides, in both direct ionization and laser postionization modes using time-of-flight mass spectrometry (TOF-MS). Desorption was performed with a 5-ns pulse width 355-nm laser beam. The molecular weights are $1141.5 \mathrm{u}$ for the cyclic decapeptide gramicidin S, 1348 $\mathrm{u}$ for the linear peptide substance $\mathrm{P}$, and $1880 \mathrm{u}$ for the linear peptide gramicidin D. Three preparatory methods for laser desorption were examined:

1. Matrix-assisted laser desorption with concomitant ionization (MAL_DI) and also MAI,D to produce the neutral molecules, by using commonly employed matrices.

2. Desorption from the neat compounds (small crystallites). 
3. Desorption from a layered arrangement by using an anthracene film as a base and then spraying about 2 $\mu \mathrm{L}$ of a dilute aqueous analyte solution onto the horizontally positioned film held at approximately $-40^{\circ} \mathrm{C}$ and then pumping on the sample to sublimate the ice film to attempt to obtain a dispersed submonolayer of the analyte (to avoid or minimize analyte-analyte interactions).

Photoionization studies of fullerenes also were performed. Raw carbon soot prepared in a standard way from a carbon arc discharge in an atmosphere of He was pressed into In foil. Pulsed (5-ns) 1064-nm laser desorption and 118-nm photoionization were used. This soot had been extracted previously with toluene at room temperature to remove significant amounts of $C_{60}$ and other lower mass fullerenes. The data here were taken at desorption laser powers (about $10^{7}$ $\mathrm{W} / \mathrm{cm}^{2}$ for 5 -ns pulses) below where direct ionization was observed, which made synthesis of the higher fullerenes by recombination in a high density plume very unlikely. This synthesis is unlikely because other studies in our laboratory show synthesis in the desorption plume, by using, for example, purified $C_{60}$ or $C_{70}$, that occurs only under laser desorption intensities where direct ions are plentiful.

All experiments were conducted in a stainless steel vacuum chamber with a typical operating pressure of $\sim 1 \times 10^{-8}$ torr. In the laser postionization experiments, neutrals desorbed from the sample were phor toionized by a focused $118-\mathrm{nm}$ laser beam that passed approximately $1 \mathrm{~mm}$ above and parallel to the sample surface. The photoions were analyzed by a reflecting time-of-flight (TOF) mass spectrometer and were detected by a dual microchannel plate assembly. In the postionization experiments, reflector potentials were adjusted so that all stable secondary ions formed at or within micrometers of the surface were transmitted through the reflector and, thus, not detected. Alternatively, positive MALDI TOF-MS spectra were recorded after minor adjustments of the mass spectrometer voltages so that these ions were reflected to the microchannel plate detector. The two-stage reflecting mass spectrometer was operated with $4-\mathrm{keV}$ drift energy, $4-\mathrm{keV}$ acceleration over about a $4-\mathrm{mm}$ gap, and approximately 2 -m effective drift length.

\section{Results}

MALDI mass spectra of the relatively small peptides and oligonucleotides are produced easily and provide high signal-to-noise ratios. For example, Figure la shows the MALDI spectrum of gramicidin $S$ when 3-hydroxypicolinic acid (3HPA) was used as a matrix [3]. There is negligible fragmentation observed for the MALDI spectra under typical operating conditions of approximately $10^{7} \mathrm{~W} / \mathrm{cm}^{2}$ irradiance and analyte-tomatrix ratios of $1: 1000$ to $1: 10,000$.

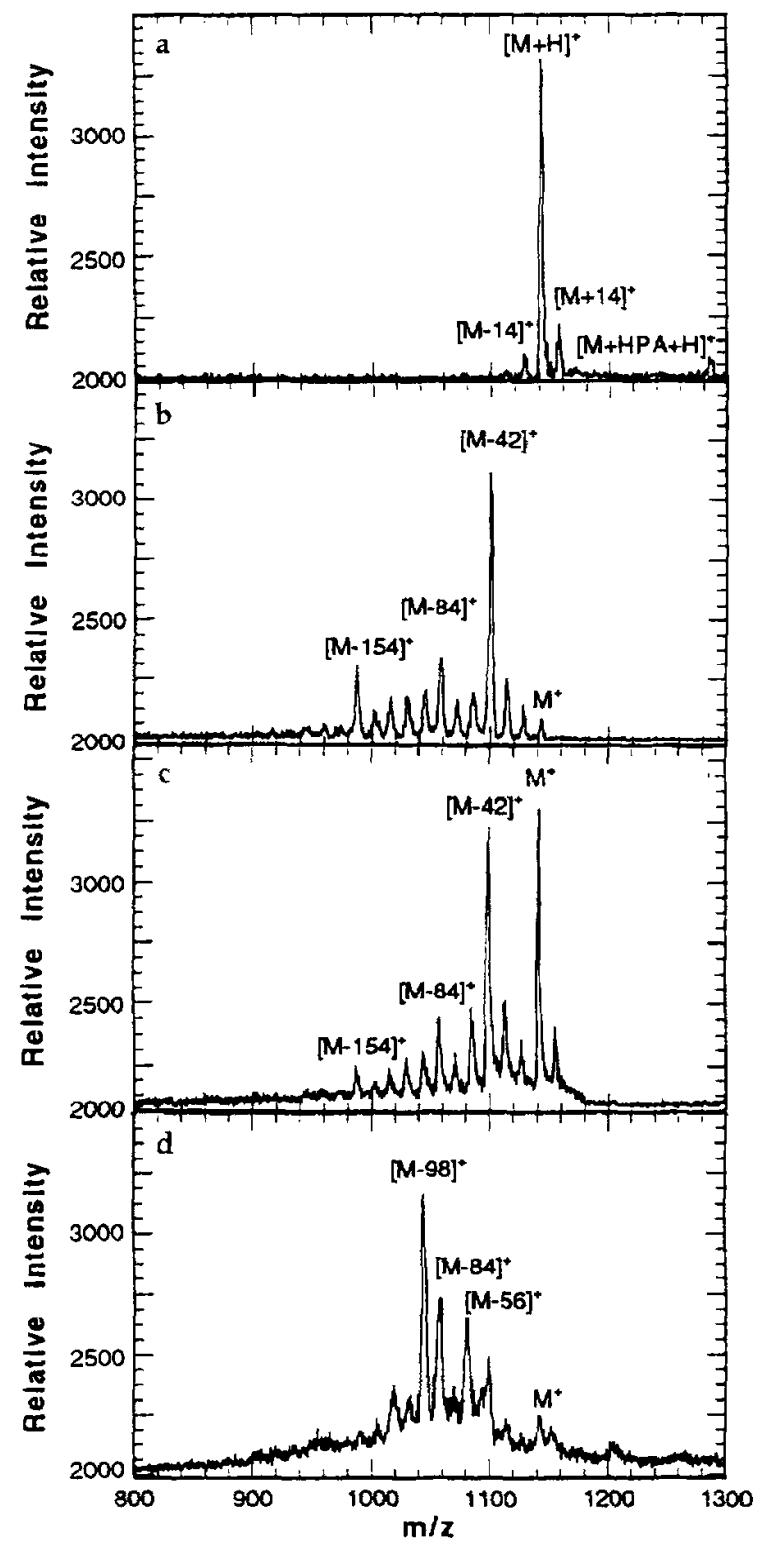

Figure 1. (a) Time-of-flight MALDI mass spectrum of gramicidin $\mathrm{S}$ with $3 \mathrm{HPA}$ as a matrix at $355 \mathrm{~nm}$ with analyte-to-matrix ratio of 1:5000. (b) 118-nm single-photon ionization TOF mass spectrum of gramicidin $S$ desorbed at $355 \mathrm{~nm}$ (approximately $10^{7}$ $W / \mathrm{cm}^{2}$ ) from a submonolayer on anthracene. (c) and (d) 118-nm single-photon ionization TOF mass spectrum of gramicidin $\mathrm{S}$ desorbed at $355 \mathrm{~nm}$ (approximately $10^{7} \mathrm{~W} / \mathrm{cm}^{2}$ ) from the matrices $3 \mathrm{HPA}$ and DHB, respectively.

Postionization was performed (or attempted) primarily by using coherent $118-\mathrm{nm}$ light $(10.5-\mathrm{eV}$ photons) generated by tripling a second 355-nm laser beam in Xe phase matched with Ar. In our experiments, parent or large fragment photoion signals could be obtained only for gramicidin $S$ and not the other two peptides or oligonucleotides. This gramicidin $S$ pho- 
toion signal could be obtained with any of the three preparation techniques; however, the layered sample on anthracene provided by far the strongest signals. Figure $1 \mathrm{~b}$ shows the spectrum of gramicidin $S$ from the layered preparation, whereas Figure $1 \mathrm{c}$ and $\mathrm{d}$ shows the data from a MALD preparation with the matrices 3HPA [3] and 2,5-dihydroxybenzoic acid (DHB) [12]. The degree of photofragmentation in Figure $1 \mathrm{~b}, \mathrm{c}$, and d suggests that the desorption from the 3 HPA produced internally cooler gramicidin $S$ molecules than for the anthracene layered arrangement which in turn produced cooler molecules than for the DHB matrix. (Note that somewhat higher irradiances are necessary for use of 3HPA relative to DHB for examination of peptides and proteins, probably due to the relative absorptivity of the molecules.) This cooler result for 3HPA could be related partially to the greater effectiveness of 3HPA versus DHB for oligonucleotides [3]. Other recent work from our laboratory has illustrated for the smaller biomolecules tryptophan and serotonin how variation of the desorption conditions readily leads to significant changes in photoion fragmentation [13].

Although a substantial amount of $\mathrm{M}^{\cdot+}$ photoion was observed, Figure $1 b, c$, and $d$ show a series of peaks due to the loss of multiples of $14 \mathrm{u}$. Occasionally a small $[M+14]^{+}$ion was observed too. Because only minor fragmentation of directly formed ions was observed for the preparation-desorption conditions, it is reasonable to conclude that these $[\mathrm{M}-n(14)]^{+}$peaks result from the 118-nm single-photon ionization. The structure of gramicidin $S$ and the continuous series of peaks with multiples of 14-u mass loss suggest that such fragmentation is mainly related to the isobutyl (from leucine) and isopropyl (from valine) side groups. It cannot be ruled out, however, that loss of some of the peptide backbone occurs; that is, one can construct possible fragmentations that involve loss of some of the peptide backbone that would produce at least some of the observed fragment masses. Note in Figure $1 \mathrm{~b}$ and $\mathrm{c}$ that the series of mass losses extends to $154 \mathrm{u}$ and beyond, which is greater than that associated with loss of a single side group, thus suggesting two and even three side-group cleavages or two and three bond cleavages of the peptide backbone. Clearly, mass loss in excess of any single side group shows multiple bond scissions-rearrangements. Also note, especially in Figure 1b and c, the noticably elevated baseline that extends to low mass in contrast to the quieter baseline above the molecular photoion; this undoubtedly represents a metastable dissociative continuum.

With regard to the fullerenes, it is apparently not difficult to observe rather large fullerene molecular photoions; sec Figure 2. The slow falloff in signal with mass in this spectrum easily could be due simply to decreasing concentrations with increasing mass and /or lower desorption yields with increasing mass. There is no obvious rapid falloff in ionization efficiency with mass seen in Figure 2.

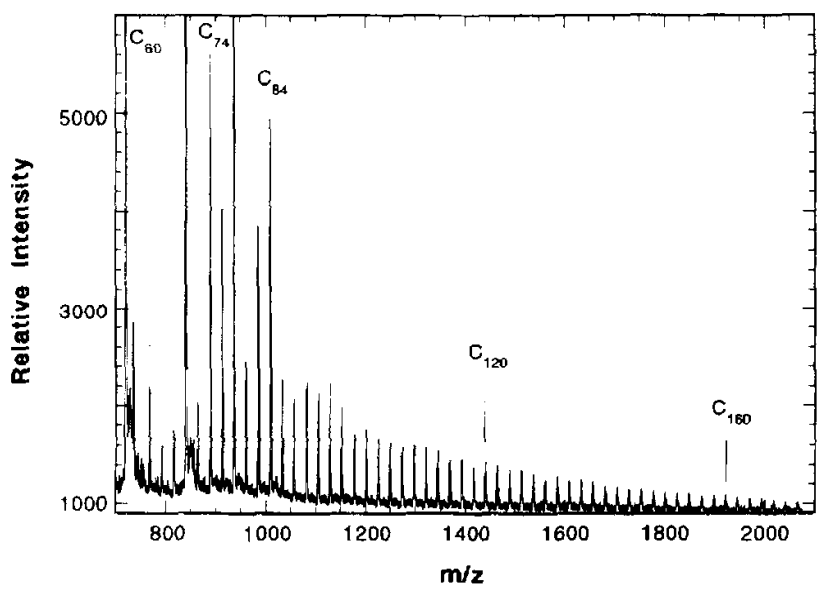

Figure 2. 118-nm single-photon ionization TOF mass spectrum of fullerenes from a pre-extracted soot, desorbed with 1064-nm laser light (approximately $10^{7} \mathrm{~W} / \mathrm{cm}^{2}$ ).

\section{Discussion}

\section{Peptides and Oligonucleotides}

The reason photoions of gramicidin $S$ were observed and gramicidin D, substance $\mathrm{P}$, or oligonucleotides were not may be due to the cyclic nature of the gramicidin $S$ molecule. It could be that backbone cleavage results for the linear molecules, but not for the cyclic peptide because of a greater structural stability for the cyclic compound. Alternatively, if backbone cleavage does readily occur even for gramicidin $S$ upon photoionization, then a nearly intact molecule (modest mass loss by some side group and/or small amount of backbone loss) still can be observed, whereas for a linear molecule, a wide range of smaller fragments would result. If many backbone dissociative channels are available for the linear peptides, which produce ions in the mass range of several hundred units and below, then another limitation would be loss of viable signal-to-noise ratio because the signal would be distributed over many channels. Facile multiple backbone cleavage could be a potential explanation for the null results for the linear peptides. Facile backbone cleavage was observed previously for even the small tripeptide Gly-Ala-Leu desorbed from water ice by a pulsed $\mathrm{CO}_{2}$ laser and ionized by 118-nm light [14].

Observations of low mass photoions from gramicidin $S$ and $D$ and substance $P$ were hindered by the large amounts of ions associated with the matrix or substrate layer and metastable decays that give rise to baseline noise. Also problematic for observation of low mass peptide fragment ions from these systems was their low concentration, which was due to the fact that the analyte had to be in very small relative abundance to ensure reasonable isolation of the analyte within the matrix crystal or on the anthracene layer. Laser desorption from crystallites of the neat peptides gives rise to easily observed low mass photoion signals. 
If our mass spectral interpretation that side group dissociation is prevalent is correct for gramicidin $S$ photoions, this makes an interesting comparison with tandem mass spectrometry results for $[\mathrm{M}+\mathrm{H}]^{+}$precursor ions. In the tandem mass spectrometry results, side group elimination is usually associated with kiloelectronvolt collision-induced dissociation (CID) and not CID at the lower energies (tens of electronvolts) associated with triple quadrupole instruments [15]. Also, recent work with surface-induced dissociation (SID) shows these side group losses at lower (20-90-eV) energies [16]. Of course SID is expected to deposit energy considerably more efficiently than CID for a given impact energy. However, this comparison with CID and SID only can be suggestive of very large internal energies in these molecules after desorption because different (odd versus even electron) precursors are involved for the photoionization experiments and the CID/SID experiments.

Perhaps a more important analogy is to the related recent work on the collisional ionization of neutral-gas phase peptides [17, 18]. For modest sized peptides (still below $1000 \mathrm{u}$ ) [18], a four-sector tandem mass spectrometer was modified with two collision cells to conduct neutralization-reionization mass spectrometry. A conclusion was reached [18] that radical molecular ions are generally not formed and the observed fragment ions tend to be of relatively low mass (extensive fragmentation).

\section{The Effect of Cooling by Entrainment in a Supersonic Jet}

Although we observed no photoion signals from these linear peptides between 1300 and $2000 \mathrm{u}$, other experimenters, who used a pulsed supersonic jet to cool the laser-desorbed peptides, have observed photoion sig nals for peptides up to about 2000 u $[5-7,19]$, including gramicidin $\mathrm{D}[7,19]$. In these cases, multiphoton ionization (MPI) was used with ultraviolet wavelengths, specifically 255 and $270 \mathrm{~nm}$ in refs 7 and 19 . respectively. The obvious conclusion is that the cooling of internal degrees of freedom is responsible for the positive results with the supersonic jet, although there also could be a wavelength effect involved because two photons at 255 or $270 \mathrm{~nm}$ represent less energy than a 118-nm photon. The negative results for peptides larger than about $2000 \mathrm{u}$ could be due to greater initial internal energy per bond and/or less efficient cooling that results in increased decomposition during photoionization. Also, as the desorption yield for peptides and other large biomolecules decreases with mass, at some point it could be just a matter of decreasing signal-to-noise ratios below the detection limit; this point should not be overlooked.

It is interesting to note that clusters of gramicidin D have been observed in some supersonic jet laser postionization experiments, with an observation of up to cight gramicidin D molecules (15,040 u) [19]. In fact, it is curious why the photoionization of such large clusters was not considered in the discussions $[9,10]$ that suggested that large molecules do not ionize efficiently.

\section{The Case of Perfluorinated Polyethers}

Recently, Anex et al. [20] reported on the laser desorption and laser postionization of large perfluorinated polyethers. In those experiments pulsed jet entrainment for cooling was employed and the oligomers were functionalized with a phenyl end group. Multiphoton ionization that included the wavelength 193 $\mathrm{nm}$ was used. The time-of-flight mass spectra were dominated by parent masses and extended to $7000 \mathrm{u}$. If the theory that larger molecules do not ionize due to competition from charge recombination is correct, then one must ask why these larger molecules are seen easily. Is it the fact that the chromophore group is at the end of the molecule? Should not some of the single-photon ionization for linear peptides and oligonucleotides, which are considerably smaller in size, occur for electron orbitals located near the chain end? Is it something about an aromatic side group? Note that the MPI of linear peptides also utilize naturally occurring aromatic side groups $[5-7,19]$.

A more obvious answer is that the perfluorinated polyethers, unlike peptides, are not very polar and have no hydrogen bond donating groups $(\mathrm{OH}$ or $\mathrm{NH})$. Thus the binding energy of the polyethers to the surface will be much lower and desorption will occur for considerably lower energy input for a given molecular weight. Thus it is likely that, in turn, the internal energy for a given molecular weight will be much lower for the polyether than the peptide or oligonucleotide, and this would translate into greater stability for the polycther for a given molecular weight, or alternatively, a higher molecular weight range accessible for the perfluorinated polyether, as observed.

\section{The Case of Fullerene Photoionization}

The electronic and geometric structure of the fullerenes such as $C_{60}$ obviously are much different than for peptides or other nonconjugated linear oligomers, although the discussion in ref 10 that describes nonprompt photoionization does not emphasize distinctions in molecular structure. Nonprompt electron emission for multiphoton ionization of fullerenes has been observed [21-23]. Intersystem crossing and/or internal conversion rates become very fast with increasing temperature for $\mathrm{C}_{60}$, and fluorescence reportedly disappears above just $350 \mathrm{~K}$ [24]. Multiphoton ionization with nanosecond-long pulses thus results in a great deal of vibrational energy deposited in the molecule, and the ionization takes on, at least in part [23], a thermionic character. However, in contrast, single-photon ionization at $118 \mathrm{~nm}$ shows only prompt emission [21]. Furthermore, our results (Figure 2) show 
no rapid falloff in ionization efficiency in contrast to the peptide parent photoions of Figure 2 of ref 9 or Figure 2 of ref 10.

\section{The Case of High Molecular Weight Solid-State Polymers}

So far we have discussed only the ionization of gasphase oligomers. However, when the issue of photoionization probabilities of oligomers and even very high molecular weight polymers (with molecular weights of $10^{5}$ to $10^{6} \mathrm{u}$ ) is considered, there really is no reason not to evaluate the situation for solid molecular samples. Substantial research has been conducted to date in the surface chemical analysis of bulk polymers by ultraviolet photoelectron spectroscopy (UPS) and $x$-ray photoelectron spectroscopy (XPS) at the solid-vacuum interface. Because at high energies the molecular density of states decreases and it has been argued that one can expect, therefore, at higher photon energies less chance for electron transfer into a state leading to charge recombination $[9,10]$, we consider in particular the lower energy range of UPS, even though most polymer analysis is typically performed by XPS. If there is difficulty in photoionization of polymeric systems due to a negligible cross section, it should be most apparent in the lower energy range of UPS. In UPS, use of a He discharge lamp that gives light at $21.2 \mathrm{eV}$ (He I emission) is common, although there are also bulk polymer studies that used $\mathrm{Ne}$ (16.8-eV photons) or $\mathrm{Ar}$ (11.7-cV photons) discharge lamps (see, e.g., $[25,26])$, and monochromaticized light down to 9 $\mathrm{eV}$ [27]. Although we are not aware of any particular study that examined secondary electron yield versus molecular weight, no difficulty in obtaining photoelectron spectra has been reported for the UPS of bulk polymers, for which there is an extensive literature; for a review, see ref 28 .

\section{Conclusions}

We conclude that there is insufficient experimental evidence to support the theory $[9,10]$ that large molecules do not photoionize. We have presented a number of contrary examples and discussions to this effect. Difficulty in observing intact molecular ions of large peptides or other polar oligomers by mass spectrometry appears to be due to enhanced fragmentation of the radical cation as a result of the increasing molecular internal energy per bond after laser desorption, whether or not a matrix is used or a cooling jet is employed in an attempt to minimize this internal excitation.

Time-of-flight single-photon ionization (SPI) mass spectra of gramicidin $\mathrm{S}$ show substantial fragmentation but with fragment ions relatively near in mass to the parent ion due to the particular stability of the cyclic structure. Null results were obtained for molecular photoions of gramicidin $\mathrm{D}$, substance $\mathrm{P}$, and oligonucleotides - molecules only slightly more massive than gramicidin S. Results from the literature where jet cooling was used show that somewhat larger peptides $(\leq 2000 \mathrm{u})$ can be photoionized intact; these peptides include large clusters of gramicidin $\mathrm{D}$ that extend to $15,040 \mathrm{u}$. Time-of-flight mass spectra of perfluorinated polyethers [20] extend to much higher masses than do peptides for similar experimental conditions which reflect the higher surface-bulk binding energies of the peptides with their significant polarities and hydrogen bonding. These higher binding energies can be expected to translate into higher internal energies per bond after desorption and thus lower stabilities for the peptide molecular radical cation. Radical molecular cations thus appear inherently much less stable than the electronically closed-shell protonated species common in MALDI, which is also in agreement with recent collisional ionization of neutral gas-phase peptides [18].

Furthermore, SPI of laser-desorbed neutral fullerenes shows a mass distribution that extends beyond $2000 \mathrm{u}$ with no evidence of rapid falloff in parent photoion yield. Finally, the literature shows that UPS spectra of very large molecular weight organic polymer solids are routinely obtained and also show no diminution in ionization efficiency as molecular size increases. Therefore, it is concluded that large molecules can photoionize efficiently, but the limitation for photoionization of gas-phase biomolecular oligomers is fragmentation, which is greatly enhanced by the considerable internal energy associated with the desorption techniques studied to date.

\section{Acknowledgments}

The authors thank Dr. Thomas Shaler for helpful discussions. Financial support from National Institutes of Health, National Center for Human Genome Research (Grant HG00174), is gratefully acknowledged.

\section{References}

1. Karas, M.; Hillenkamp, F. Anal. Chem. 1988, 60, 2299.

2. Chait, B. T.; Kent, S. B. H. Science 1992, 257, 1885.

3. Wu, K. J.; Steding A.; Becker, C. H. Rapid Commun. Mass Spectrom. 1993, 7, 142

4. Schieltz, D. M.; Chou, C.-W.; Luo, C.-W.; Thomas, R. M.; Williams, P. Rapid. Commun. Mass Spectrom. 1992, 6, 631.

5. Dey, M.; Böhm, W.; Prinke, S.; Grotemeyer, J. Proceedings of the 40th American Society for Mass Spectrometry Conference, 1992, p 332.

6. Frey, R.; Holle, A.; Mayer, F. J.; Schäfer, R. Proceedings of the 40th American Society for Mass Spectrometry Conference, 1992, p 334.

7. Kinsel, G. R.; Lindner, J.; Grotemeyer, J. Org. Mass Spectrom. 1991, 26, 1052.

8. Huth-Fehre, T.; Becker, C. H. Rapid, Commun. Mass Spectrom. $1991,5,378$.

9. Schlag, F. W.; Grotemeyer, J.; Levine, R. D. Chem. Phys. Lett. 1992, 190, 521 . 
10. Schlag, E. W.; Levine, R. D. J. Phys. Chem. 1992, 96, 10608.

11. Beavis, R. C.; Chait B. T. In Methods and Mechanisms for Producing lons from Large Molecules, Standing, K. G.; Ens, W., Eds.; Plenum Press: New York, 1991; p 227.

12. Strupat, K.; Karas, M; Hillenkamp, F. Int. /. Mass Spectrom. Ion Processes 1991, 111, 89.

13. Ayre, C. R.; Moro, L.; Becker, C. H. Anal. Chem. 1994, 66, 1610.

14. Becker, C. H.; Jusinski, L. E.; Moro, L. Int. I. Mass Spectrom Ion Processes 1990, 95, R1.

15. Johnson, R S.; Martin, S. A.; Biemann, K.; Stults, J. T.; Watson, J. T. Anal. Chem. 1987, 59, 2621.

16. McCormack, A. L.; Somogyi, A.; Dongré, A R.; Wysocki, V. H. Anal. Chem. 1993, 65, 2859

17. Cordero, M. M.; Houser, J. J.; Wesdemiotis, C. Anal. Chem 1993, 65, 1594

18. Wu, A.; Fenselau, C. Org. Mass Spectrom. 1993, 28, 1034.
19. Köster, C.; Grotemeyer, J.; Schlag, E. W. Z. Naturforsch. 1990, $45 a, 1285$

20. Anex, D. S.; de Vries, M. S.; Knebelkamp, A.; Bargon, J.; Wendt, H. R.; Hunziker, H. E. Int. J. Mass Spectrom. Ion Processes 1994, 131, 319.

21. Wurz, P.; Lykke, K. R. J. Chem. Phys. 1991, 95, 7008.

22. Wur, P.; Lykke, K. R. J. Phys. Chem. 1992, 96, 10129.

23. Ding, D.; Compton, R. N.; Haufler, R. E.; Klots, C. E. J. Phys. Chem. 1993, 97, 2500.

24. Haynes, D. R.; Tokmakoff, A.; George, S. M. Chem. Phys. Lett. 1993, 214, 50 .

25. Asada, S.; Seki, K.; Inokuchi, H. Chem. Phys. Lett. 1986, 130, 155.

26. Chen, S.-X.; Chen, L.-S.; Seki, K.; Sato, N.; Inokuchi, H. Chinese Sci. Bull. 1989, 34, 636.

27. Fuihira, M.; Inokuchi, H. Chem. Phys. Lett. 1972, 17, 554.

28. Salaneck, W. R. Crit. Rev. Solid State Mat. Sci. 1985, 12, 267. 